\title{
A Deep Eutectic Solvent as an Extraction Solvent to Separate and Preconcentrate Parabens in Water Samples Using in situ Liquid-Liquid Microextraction
}

\author{
Dandan Ge, ${ }^{\circledR * a}$ Ying Wang, ${ }^{a}$ Qian Jiang ${ }^{a}$ and Enrui Dai ${ }^{a}$ \\ ${ }^{a}$ Department of Chemical Science \& Technology, Kunming University, 650214 Kunming, China
}

\begin{abstract}
In this study, an in situ deep eutectic solvent-liquid-liquid microextraction method together with high performance liquid chromatography was developed for the determination of parabens in aqueous sample. The hydrophobic deep eutectic solvent, formed in situ by the DL-menthol and decanoic acid was used as the extraction solvent. Various parameters affecting the extraction efficiency including the composition of deep eutectic solvent, temperature of sample solution, extraction time, solution $\mathrm{pH}$ and salt concentration were investigated and optimized. Under the optimum conditions, the linear range of calibration curves was in the range of 3-1000 $\mathrm{ng} \mathrm{mL}^{-1}$. The limits of detection and limits of quantification were in the range of 0.6-0.8 and 2.0-2.5 ng mL-1, respectively. The relative standard deviations were less than $7.2 \%$ for both intra-day and inter-days analysis. Finally, the proposed method was successfully applied to determine four parabens in environmental water samples with acceptable recoveries.
\end{abstract}

Keywords: deep eutectic solvent, in situ liquid-liquid microextraction, parabens, water analysis

\section{Introduction}

Parabens are a family of alkyl esters of $p$-hydroxybenzoic acid. They are commonly used as preservatives to protect daily-use products such as food products, personal care products and pharmaceutical products from bacterial and fungal attack. ${ }^{1,2}$ Due to the widespread use of parabens, the removal capacity of wastewater treatment plants (WWTPs) is overloaded. Thus, parabens can enter the environment through WWTPs. Besides this, some of the parabens are introduced to environment via human aquatic leisure. ${ }^{3}$ Several studies have reported that parabens can cause endocrine disruption, leading to reproductive problems and cancer. ${ }^{4,5}$ Therefore, the development of a sensitive method for the determination of parabens in environmental samples is of great importance.

In the analysis of parabens in complex matrices, a separation/enrichment method prior to instrumental analysis is required. ${ }^{6}$ Liquid-liquid extraction (LLE) as a liquid-based sample preparation method is commonly used to preconcentrate parabens. ${ }^{7,8}$ However, LLE has disadvantages such as being time consuming and requiring moderate to large amounts of costly toxic organic solvents. Therefore, the development of miniaturized and solvent-minimized sample preparation techniques has

*e-mail: dandangeiris@163.com gained increasing research interest. Currently popular approaches are minimized LLE such as single drop microextraction (SDME), ${ }^{9}$ hollow fiber protected liquidphase microextraction ${ }^{10}$ and dispersive liquid-liquid microextraction (DLLME). ${ }^{11,12}$

DLLME is a microextraction method with a ternary component solvent system. The method has been widely used to extract analytes from various matrices. ${ }^{11-14}$ The challenge associated with this method is the use of highly toxic, volatile and environmentally hazardous solvents such as 1,1,2,2-tetrachloroethane, carbon tetrachloride or chloroform as the extraction solvents. ${ }^{13,14}$ To reduce environmental burden in conventional DLLME and to answer the requirements of green chemistry, recent studies have focused on developing green, eco-friendly and cheap solvents. As a result, deep eutectic solvents (DESs) as a new type of green solvent have been introduced. ${ }^{15,16}$

DESs are defined as mixtures of two or more safe, cheap and biodegradable components, which form liquids with melting points far below those of the individual components due to the self-association effect. ${ }^{17}$ DESs are generally prepared by mixing a hydrogen bond acceptor (HBA) such as choline chloride and a hydrogen bond donor (HBD) such as phenols, carboxylic acids, sugars, urea or polyalcohols in specific molar ratios. ${ }^{15-21}$ They are considered as a new family of ionic liquids (ILs) due to their similar physical properties. ${ }^{18,19}$ Compared with 
ILs, DESs own some inimitable features such as low cost, eco-friendly, easy preparation with high purity and biodegradability. These novel properties characterize DESs as being different from other solvents used in DLLME and endow the solvents with the potential to extract trace analytes from different matrices. The use of DESs in the separation and preconcentration of organic compounds and metal ions has been reported. ${ }^{22-30}$ However, most of the early DESs used in water-containing samples have limitation as they are hydrophilic. Hence, the preparation and use of hydrophobic DESs in sample preparation methods have attracted significant attention. Zhu et al. ${ }^{31}$ reported the use of a hydrophobic DES by mixing tetrabutylammonium chloride and octanoic acid in a liquid-liquid microextraction (LLME) of synthetic pigments in beverages. In particular, Florindo et al. ${ }^{32}$ synthesized a type of DL-menthol-based hydrophobic DESs and applied to LLE of pesticides from aqueous samples. In our previous work, ${ }^{33}$ a DLmenthol-based DES was used to air-assisted DLLME of six benzophenone-type UV filters from aqueous samples. In situ LLME based on DES (in situ DES-LLME) is a mode of DLLME, in which upon addition of HBA and HBD to an aqueous solution, the formation of a DES and extraction can occur simultaneously in a single extraction step. Compared to conventional DLLME, this method owns the advantages of short extraction time, simplicity and no use of a dispersive solvent. Recently, a DES (choline chloride and $p$-chlorophenol at a 1:2 molar ratio) was simultaneously formed and used for LLME of polycyclic aromatic hydrocarbons from aqueous samples. ${ }^{34}$

In this study, an in situ DES-LLME method was developed to extract parabens in environmental water samples followed by high-performance liquid chromatography (HPLC)-diode array detector (DAD) analysis. For this purpose, two components of a DES, DL-menthol and decanoic acid, were added to an aqueous sample and then heated. The resulted solution was manually stirred during heating to ensure complete formation of the DES and dispersion of the DES into the aqueous phase. Parameters affecting the extraction efficiency were carefully optimized. Finally, the developed methods were successfully applied for the determination of parabens in environmental water samples.

\section{Experimental}

\section{Chemicals and materials}

The studied parabens, including methylparaben (MP; 99\%), ethylparaben (EP; 99\%), propylparaben (PP; 99\%), butylparaben (BP; 99\%), DL-menthol (98\%), octanoic acid (99\%), decanoic acid (98\%) and dodecanoic acid (98\%) were purchased from Aladdin Reagent Corporation (Shanghai, China). HPLC-grade methanol was bought from Merck (Darmstadt, Germany). Sodium chloride $(\mathrm{NaCl})$, hydrochloric acid $(\mathrm{HCl})$ and sodium hydroxide $(\mathrm{NaOH})$ were purchased from Merck (Darmstadt, Germany). Ultrapure water was produced on a Milli-Q water purification system (Millipore, Billerica, MA, USA). A stock solution containing the four parabens was prepared in methanol at $1 \mathrm{mg} \mathrm{mL}^{-1}$ and stored at $4{ }^{\circ} \mathrm{C}$ until use. Working solutions were prepared daily by proper dilution of the stock solution with ultrapure water.

\section{Samples}

Water samples analyzed by the proposed method were collected from different sources including tap, river, lake and wastewater in Kunming, China. Tap water was obtained from the Kunming University, China. The waste effluent water was collected from the sewage treatment plant of the Kunming University, China. The natural water sampling locations include Baoxiang River and Dianchi Lake, Yunnan province, China. The quantity of water collected is $2 \mathrm{~L}$. The natural water samples were collected at a depth of approximately $0.4 \mathrm{~m}$ below the water surface. The samples were previously filtered and stored in amber glass containers at $4{ }^{\circ} \mathrm{C}$ until analysis.

\section{Instrumentation}

Chromatographic analysis was carried out on an Agilent 1260 Infinity HPLC system (Palo Alto, CA, USA) equipped with an automated sample injector, a quaternary pump and a DAD. The injection volume was $10 \mu \mathrm{L}$. The separation was performed on a Zorbax SB- $\mathrm{C}_{18}$ column $\left(150 \times 4.6 \mathrm{~mm}^{2}\right.$ i.d., $5 \mu \mathrm{m}$ particle size, Agilent, Palo Alto, CA, USA). The gradient elution mode was used with a flow rate at $0.8 \mathrm{~mL} \mathrm{~min}^{-1}$. A mixture of water (A) and methanol (B) was used as mobile phase. The elution profile was as follows: started with 35\% B, 0-10 min B increased to $70 \%$ and kept isocratic until $17 \mathrm{~min}, 17-20 \mathrm{~min} B$ decreased to the initial condition. The detection wavelength was set at $257 \mathrm{~nm}$.

\section{Microextraction procedure}

To carry out in situ DES-LLME, $8 \mathrm{~mL}$ of an aqueous solution was transferred into a $15 \mathrm{~mL}$ glass centrifuge tube. Then, $0.09 \mathrm{~g}$ DL-menthol and $0.05 \mathrm{~g}$ decanoic acid (at a molar ratio of 2:1) were added to the aqueous solution and the mixture was immersed in a thermostated water 
bath at $70{ }^{\circ} \mathrm{C}$. The tube was manually stirred to ensure complete formation of the DES and dispersion of the DES into the aqueous phase. Consequently, a white turbid solution containing fine droplets of the DES gradually formed. After centrifugation at $4000 \mathrm{rpm}$ for $5 \mathrm{~min}$, a liquid was formed at the upper phase of the tube. Then the supernatant was collected and injected into the HPLC system for analysis.

\section{Results and Discussion}

\section{Effect of the component of DES}

The component of HBA and HBD is one of the important factors influencing the formation and extraction efficiency of a DES. In this study, DL-menthol was selected as the HBA to prepare DESs with 3 different aliphatic acids chosen as HBDs at different molar ratios. A summary of the component and molar ratios of DESs (DES 1-6) is presented in Table 1. The molar ratios of the DESs were selected according to previous studies. ${ }^{32,33}$ All the DESs were prepared as homogeneous liquids and retained upon cooling in an aqueous solution. As shown in Figure 1, higher peak areas of EP, PP and BP were obtained by DES-1 (DL-menthol and octanoic acid), followed by DES-2 (DL-menthol and decanoic acid) and DES-3 (DL-menthol and dodecanoic acid). However, an interfering peak of MP was observed when DES-1 was used as the extraction solvent. Hence, a DES composed of DL-menthol and decanoic acid was selected for further optimization.

\section{Characterization of DES}

The main force for the formation of DES is intermolecular hydrogen bonding between DL-menthol and aliphatic acids. To illustrate the interaction, Fourier transform infrared (FTIR) spectra of DL-menthol, decanoic acid and DES-2 were investigated. As shown in Figure 2, the broad band

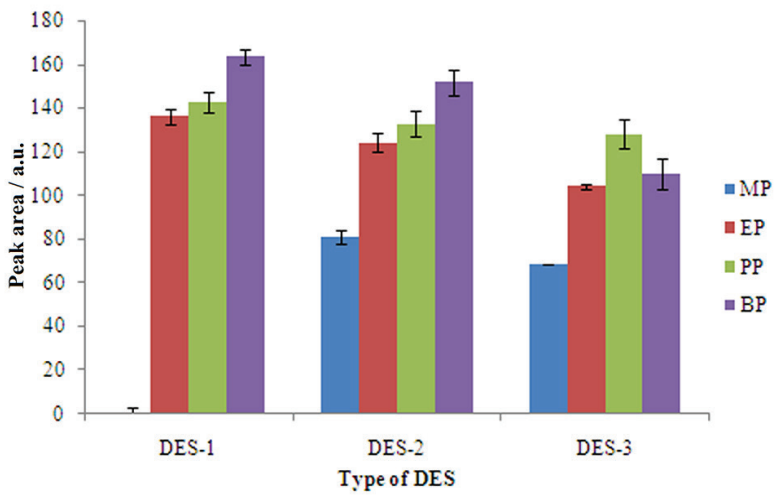

Figure 1. Effect of the type of DES on the extraction efficiency of the developed method. Extraction conditions: concentration of the analytes, $80 \mathrm{ng} \mathrm{mL}^{-1}$; aqueous solution volume, $8 \mathrm{~mL}$; aqueous phase temperature, $70{ }^{\circ} \mathrm{C}$; heating time, $7 \mathrm{~min}$; solution $\mathrm{pH}$, not adjusted; salt concentration, $0 \mathrm{mg} \mathrm{mL}{ }^{-1}$; three replicate experiments were conducted.

at $3476 \mathrm{~cm}^{-1}$ related to $\mathrm{O}-\mathrm{H}$ vibrations of decanoic acid shifted to $3420 \mathrm{~cm}^{-1}$ in the FTIR spectrum of DES-2. The possible reason is that the transfer of oxygen atom cloud point to hydrogen bond results in a slightly decrease in force constant. Hence, the change of wavelength number of the $\mathrm{O}-\mathrm{H}$ group in the DES-2 compared to decanoic acid indicates the existence of hydrogen bonding and formation of DES.

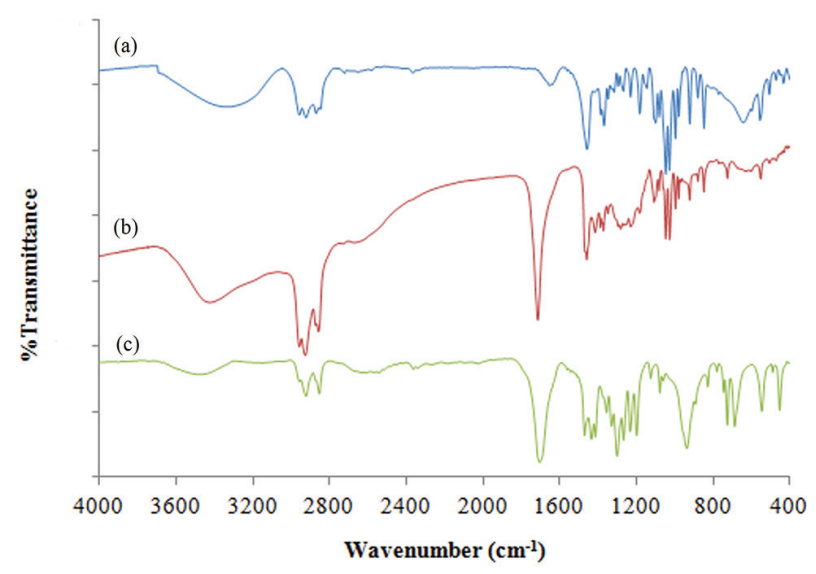

Figure 2. FTIR spectra of (a) DL-menthol, (b) DES-2, and (c) decanoic acid.

Table 1. Preparation of different hydrophobic DES

\begin{tabular}{|c|c|c|c|c|}
\hline Abbreviation & HBA & HBD & Molar ratio & Aspect in the aqueous solution \\
\hline DES-1 & \multirow{6}{*}{ DL-menthol } & octanoic acid & $1: 1$ & \multirow{6}{*}{ transparent liquid } \\
\hline DES-2 & & decanoic acid & $1: 1$ & \\
\hline DES-3 & & dodecanoic acid & $2: 1$ & \\
\hline DES-4 & & decanoic acid & $3: 1$ & \\
\hline DES-5 & & decanoic acid & $2: 1$ & \\
\hline DES-6 & & decanoic acid & $1: 2$ & \\
\hline
\end{tabular}

HBA: hydrogen bond acceptor; HBD: hydrogen bond donor; DES: deep eutectic solvent. 


\section{Effect of the molar ratio of HBA and HBD}

The molar ratio between HBA and HBD has a remarkable effect on the physicochemical properties of a DES. To investigate the effect of HBA to HBD molar ratio on the extraction efficiency, DESs with DL-menthol to decanoic acid molar ratio changing from 3:1 to 1:2 were prepared and investigated. As displayed in Figure 3, DES-5 with DL-menthol to decanoic acid at a molar ratio of 2:1 exhibited higher peak areas. Therefore, DES-5 was chosen as the extraction solvent in the following experiments.

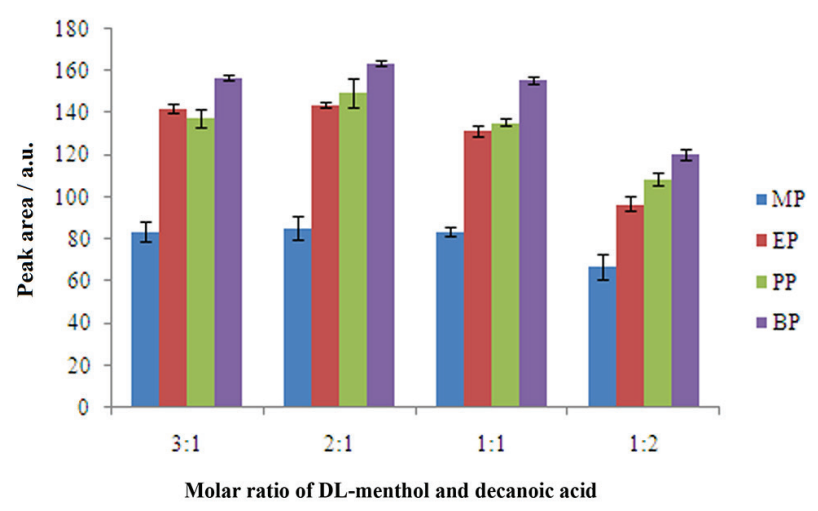

Figure 3. Effect of molar ratio of DES-5 on the extraction efficiency of the developed method. Extraction conditions: concentration of the analytes, $80 \mathrm{ng} \mathrm{mL}^{-1}$; aqueous solution volume, $8 \mathrm{~mL}$; aqueous phase temperature, $70^{\circ} \mathrm{C}$; heating time, $7 \mathrm{~min}$; solution $\mathrm{pH}$, not adjusted; salt concentration, $0 \mathrm{mg} \mathrm{mL}{ }^{-1}$; three replicate experiments were conducted.

\section{Effect of the amounts of HBA and HBD}

The extraction solvent volume has a significant influence on the extraction efficiency of LLME. In this study, by changing the amounts of DL-menthol and decanoic acid (keeping the molar ratio at 2:1), different volumes of DES could be obtained. Therefore, the extraction solvent volume was determined by the amounts of DL-menthol and decanoic acid. To study the effect of the amounts of DL-menthol and decanoic acid, different aqueous solutions containing 0.03:0.02, 0.06:0.03, 0.09:0.05 and 0.12:0.07 $\left(\mathrm{g} \mathrm{g}^{-1}\right)$ of DL-menthol and decanoic acid were prepared. According to the obtained results, the peak areas increased until 0.09:0.05 $\left(\mathrm{g} \mathrm{g}^{-1}\right)$ DL-menthol:decanoic acid and decreased thereafter. The reason for this phenomenon is that different volumes of DES-5 were obtained with different amounts of DL-menthol and decanoic acid. The formed DES-5 volume at the upper phase of the tube was 50, 107, 130 and $150 \mu \mathrm{L}$ for 0.03:0.02, 0.06:0.03, 0.09:0.05 and 0.12:0.07 ( $\left.\mathrm{g} \mathrm{g}^{-1}\right)$ of DL-menthol and decanoic acid, respectively. Figure S1 (Supplementary Information section) shows the effect of amount of DL-menthol and decanoic acid (volume of DES-5) on the extraction efficiency. Subsequently, 0.09:0.05 $\left(\mathrm{g} \mathrm{g}^{-1}\right)$ of DL-menthol and decanoic acid was chosen for additional studies.

\section{Effect of solution temperature and heating time}

DES-5 was prepared by mixing DL-menthol and decanoic acid throughout heating. Therefore, the formation of a DES is related to heating temperature and heating time. Furthermore, temperature is a parameter that may influence the viscosity of a DES and diffusion coefficients of the analytes. Hence, the effect of solution temperature was investigated from 50 to $80{ }^{\circ} \mathrm{C}$. The obtained results showed that the peak areas of all analytes reached their perspective maxima at the temperature of $60{ }^{\circ} \mathrm{C}$ and decreased slightly thereafter. The increase of temperature can increase the transfer analytes into the extraction solvent and at the same time enhance the migration of analytes out of the extraction solvent. Thus, the peak areas varied at different temperatures. Furthermore, the formation of DES was not complete at temperatures $<60{ }^{\circ} \mathrm{C}$. Therefore, the temperature of sample solution was determined to be $60^{\circ} \mathrm{C}$.

The influence of heating time on the extraction of target analytes was examined in the range of 1-9 $\mathrm{min}$. It was found that the peak areas increased up to $5 \mathrm{~min}$ and remained nearly constant with additional heating time. Hence, the heating time was selected to be $5 \mathrm{~min}$ for the subsequent experiments.

\section{Effect of solution $\mathrm{pH}$}

The solution $\mathrm{pH}$ affects the existing forms of the analytes and DES formation. To evaluate the effect of solution $\mathrm{pH}$ on the extraction efficiency, solution $\mathrm{pH}$ was adjusted in the range of 3-11 by $0.1 \mathrm{M} \mathrm{HCl}$ or $\mathrm{NaOH}$. It can be seen from Figure 4 that at $\mathrm{pH}$, the highest peak areas

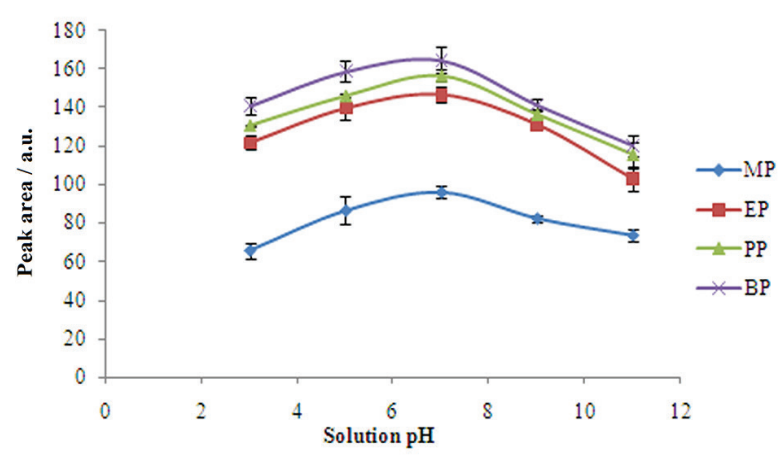

Figure 4. Effect of solution $\mathrm{pH}$ on the extraction efficiency of the developed method. Extraction conditions: concentration of the analytes, $80 \mathrm{ng} \mathrm{mL} \mathrm{mL}^{-1}$; aqueous solution volume, $8 \mathrm{~mL}$; DL-menthol:decanoic acid amount, 0.09:0.05; aqueous phase temperature, $60{ }^{\circ} \mathrm{C}$; heating time, $5 \mathrm{~min}$; salt concentration, $0 \mathrm{mg} \mathrm{mL}^{-1}$; three replicate experiments were conducted. 
were obtained. However, at solution $\mathrm{pH}$ values of 3,9 and 11, the obtained DES volumes decreased, and lower peak areas were achieved. These results showed that the solution $\mathrm{pH}$ affected the formation of DES. It should be noted that the $\mathrm{pH}$ values of all samples were around 7 . Hence, the solution $\mathrm{pH}$ was not adjusted in this study.

\section{Effect of salt addition}

The addition of a salt decreases the solubility of analytes in the aqueous solution (salting-out effect), and thus the extraction efficiency could be improved. The effect of salt addition on the proposed method was investigated over a $\mathrm{NaCl}$ concentration range of $0-10 \%$ $(\mathrm{m} / \mathrm{v})$, and the results are shown in Figure 5. According to the obtained results, the peak areas initially increased until $1 \%$ of $\mathrm{NaCl}$ and decreased gradually by increasing the concentration of $\mathrm{NaCl}$. The observation may be attributed to an increase in the viscosity of the sample solution that led to a decrease in the mass transfer rates of the analytes into the aqueous solution. Hence, $1 \%(\mathrm{~m} / \mathrm{v})$ as optimum salt concentration was selected for further experiments.

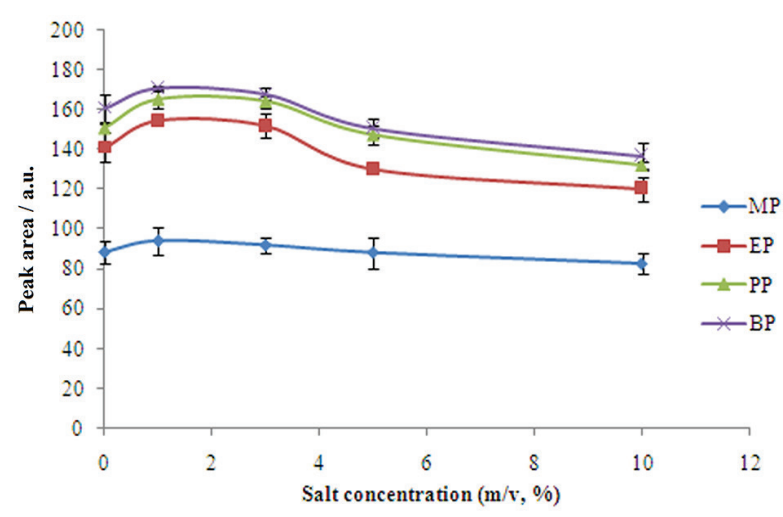

Figure 5. Effect of salt concentration on the extraction efficiency of the developed method. Extraction conditions: concentration of the analytes, $80 \mathrm{ng} \mathrm{mL}^{-1}$; aqueous solution volume, $8 \mathrm{~mL}$; DL-menthol:decanoic acid amount, 0.09:0.05; aqueous phase temperature, $60^{\circ} \mathrm{C}$; heating time, $5 \mathrm{~min}$; solution $\mathrm{pH}$, not adjusted; three replicate experiments were conducted.

\section{Analytical performance}

The analytical performance of the proposed method was evaluated in terms of linear range, limit of detection (LOD), limit of quantification (LOQ), extraction recoveries (ER) and precision. The obtained results are listed in Table 2. To define the linear range, 7 calibration points were used. The calibration curves were linear in the range of 3-1000 $\mathrm{ng} \mathrm{mL}^{-1}$ with satisfactory correlation coefficients $(r>0.9961)$. The LODs (signal-to-noise ratio of 3) ranged from 0.6 to $0.8 \mathrm{ng} \mathrm{mL}^{-1}$ and the LOQs (signal-to-noise ratio of 10) ranged from 2.0 to $2.5 \mathrm{ng} \mathrm{mL}^{-1}$. The intra-day and interdays precisions of the method were tested using a solution with a concentration of $10 \mathrm{ng} \mathrm{mL}^{-1}$ being extracted with the developed method in a day $(n=6)$ and for five consecutive days, respectively. The obtained relative standard deviations (RSDs) were less than $7.2 \%$ for intra-day and inter-days determinations. The ER (defined as the amount of analytes extracted divided by total amount of analytes in the sample, $\mathrm{ER}(\%)=\mathrm{C}_{\mathrm{DES}} \mathrm{V}_{\mathrm{DES}} / \mathrm{Caq}$ Vaq $\times 100$, where $\mathrm{C}_{\mathrm{DES}}, \mathrm{V}_{\mathrm{DES}}$, $\mathrm{Caq}$, Vaq are the analyte concentration in DES after extraction, the final volume of DES phase after extraction, the concentration of the analyte in the aqueous sample, and aqueous sample volume, respectively) were evaluated at 10 and $100 \mathrm{ng} \mathrm{mL}^{-1}$ under the optimized conditions. ER in the range of 69.1 and $78.5 \%$ were obtained. Based on these results, the proposed method has the potential to determine parabens in aqueous samples.

\section{Analysis of real samples}

The applicability of the proposed method was evaluated in determination of the parabens in water samples including tap, river, lake and wastewater. As shown in Table 3, no analytes were detected in tap water, river water and lake water samples, and MP was detected at $3.5 \mathrm{ng} \mathrm{mL}^{-1}$ in wastewater sample. This is a common observation that has been reported in literature for the detection of parabens due to frequent use of MP as preservative..$^{35}$ The presence

Table 2. Analytical performance of the proposed method for the determination of parabens in aqueous samples

\begin{tabular}{|c|c|c|c|c|c|c|c|c|}
\hline \multirow{2}{*}{ Analyte } & \multirow{2}{*}{$\begin{array}{l}\text { Linearity range / } \\
\quad\left(\text { ng mL } \mathrm{m}^{-1}\right)\end{array}$} & \multirow{2}{*}{$\mathrm{r}$} & \multirow{2}{*}{$\begin{array}{c}\text { LOD / } \\
\left(\mathrm{ng} \mathrm{mL}^{-1}\right)\end{array}$} & \multirow{2}{*}{$\begin{array}{c}\text { LOQ / } \\
\left(\mathrm{ng} \mathrm{mL}^{-1}\right)\end{array}$} & \multicolumn{2}{|c|}{$\mathrm{RSD} / \%$} & \multicolumn{2}{|c|}{$\mathrm{ER} / \%$} \\
\hline & & & & & Intra-day & Inter-day & $10 \mathrm{ng} \mathrm{mL}^{-1}$ & $100 \mathrm{ng} \mathrm{mL}^{-1}$ \\
\hline MP & $3-1000$ & 0.9985 & 0.8 & 3.1 & 4.6 & 2.5 & 69.1 & 71.4 \\
\hline EP & $3-1000$ & 0.9961 & 0.6 & 2.5 & 5.4 & 3.5 & 76.0 & 74.6 \\
\hline PP & $3-1000$ & 0.9989 & 0.6 & 2.5 & 3.4 & 7.2 & 78.5 & 76.1 \\
\hline BP & $3-1000$ & 0.998 & 0.8 & 3.1 & 1.1 & 3.6 & 72.2 & 73.4 \\
\hline
\end{tabular}

r: correlation coefficient; LOD: limit of detection; LOQ: limit of quantification; RSD: relative standard deviation; ER: extraction efficiency; MP: methylparaben; EP: ethylparaben; PP: propylparaben; BP: butylparaben. 
Table 3. Analytical results for the determination of parabens in real water samples

\begin{tabular}{|c|c|c|c|c|c|c|c|c|c|}
\hline \multirow[b]{2}{*}{ Analyte } & \multirow[b]{2}{*}{$\begin{array}{l}\text { Spiked level / } \\
\left(\mathrm{ng} \mathrm{mL}^{-1}\right)\end{array}$} & \multicolumn{2}{|c|}{ Tap water } & \multicolumn{2}{|c|}{ Lake water } & \multicolumn{2}{|c|}{ River water } & \multicolumn{2}{|c|}{ Domestic wastewater } \\
\hline & & $\begin{array}{c}\text { Detected } \\
\text { level / } \\
\left(\mathrm{ng} \mathrm{mL^{-1 } )}\right.\end{array}$ & $\begin{array}{c}\mathrm{RR} \pm \mathrm{RSD} / \\
\%\end{array}$ & $\begin{array}{c}\text { Detected } \\
\text { level / } \\
\left(\mathrm{ng} \mathrm{mL} \mathrm{mL}^{-1}\right)\end{array}$ & $\begin{array}{c}\mathrm{RR} \pm \mathrm{RSD} / \\
\%\end{array}$ & $\begin{array}{c}\text { Detected } \\
\text { level / } \\
\left(\mathrm{ng} \mathrm{mL}^{-1}\right)\end{array}$ & $\begin{array}{c}\mathrm{RR} \pm \mathrm{RSD} / \\
\%\end{array}$ & $\begin{array}{c}\text { Detected } \\
\text { level / } \\
\left(\mathrm{ng} \mathrm{mL^{-1 } )}\right.\end{array}$ & $\begin{array}{c}\mathrm{RR} \pm \mathrm{RSD} / \\
\%\end{array}$ \\
\hline \multirow{3}{*}{ MP } & 0 & n.d. & - & n.d. & - & n.d. & - & 3.5 & $93.7 \pm 3.0$ \\
\hline & 4 & 4.0 & $100.7 \pm 1.0$ & 3.8 & $95.4 \pm 2.3$ & 3.7 & $92.3 \pm 2.7$ & 7.7 & $102.3 \pm 5.8$ \\
\hline & 100 & 95.8 & $95.8 \pm 7.9$ & 85.2 & $85.2 \pm 2.5$ & 89.0 & $89.0 \pm 3.3$ & 99.5 & $96.1 \pm 3.1$ \\
\hline \multirow{3}{*}{ EP } & 0 & n.d. & - & n.d. & - & n.d. & - & n.d. & - \\
\hline & 4 & 3.9 & $96.3 \pm 5.2$ & 3.8 & $95.4 \pm 1.2$ & 3.9 & $98.5 \pm 3.4$ & 3.9 & $98.7 \pm 5.9$ \\
\hline & 100 & 108.7 & $108.7 \pm 7.6$ & 95.6 & $95.6 \pm 3.5$ & 90.2 & $90.2 \pm 3.6$ & 92.4 & $92.4 \pm 0.5$ \\
\hline \multirow{3}{*}{ PP } & 0 & n.d. & - & n.d. & - & n.d. & - & n.d. & - \\
\hline & 4 & 3.8 & $95.7 \pm 2.5$ & 4.0 & $99.5 \pm 4.9$ & 4.1 & $101.4 \pm 2.0$ & 3.9 & $97.7 \pm 5.4$ \\
\hline & 100 & 100.1 & $100.1 \pm 0.9$ & 96.7 & $96.7 \pm 2.1$ & 94.6 & $94.6 \pm 1.8$ & 98.2 & $98.2 \pm 0.6$ \\
\hline \multirow{3}{*}{ BP } & 0 & n.d. & - & n.d. & - & n.d. & - & n.d. & - \\
\hline & 4 & 3.8 & $96.1 \pm 2.4$ & 3.9 & $97.7 \pm 0.4$ & 3.7 & $91.1 \pm 2.3$ & 4.0 & $99.4 \pm 2.7$ \\
\hline & 100 & 84.8 & $88.8 \pm 1.2$ & 105.8 & $105.9 \pm 3.6$ & 93.4 & $93.4 \pm 3.1$ & 98.2 & $98.2 \pm 0.7$ \\
\hline
\end{tabular}

RR: relative recovery; RSD: relative standard deviation; MP: methylparaben; n.d.: not detected; EP: ethylparaben; PP: propylparaben; BP: butylparaben.

of MP was confirmed by comparing the chromatogram of a standard and a wastewater sample (Figure 6). In order to test genuine matrix effect, the relative recoveries (RR) from samples spiked with the analytes at two concentration levels (4 and $\left.100 \mathrm{ng} \mathrm{mL}^{-1}\right)$ are presented in Table 3. RR between 84.8 to $108.7 \%$ with RSDs from 0.4 and $7.9 \%$
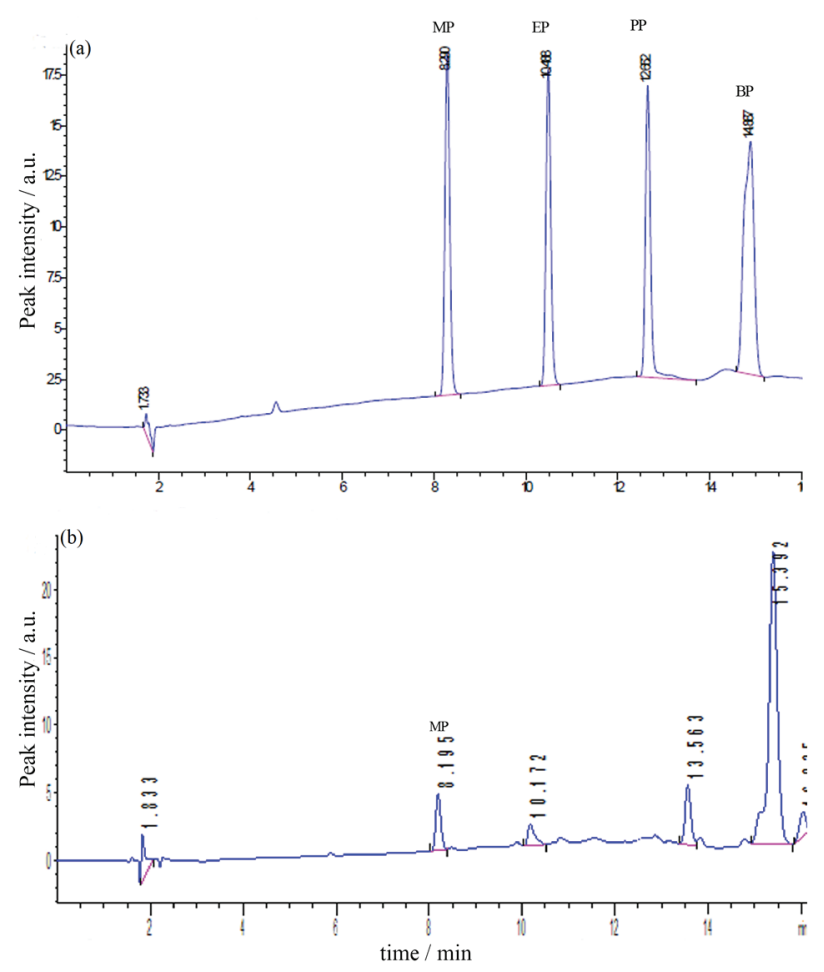

Figure 6. Chromatogram of (a) paraben standards and (b) a wastewater sample extracted by the developed method. were achieved, which show that the matrices of the samples have no significant effect on the proposed method. The developed method is feasible for determination of the parabens in water samples.

Comparison of the proposed method with other reported methods

The performance of the proposed method in combination with HPLC for determination of parabens was compared with other reported methods, considering LOD, extraction time, extraction solvent and RSD, as listed in Table 4. In general, the proposed method provided similar or better LODs and linear range, except for micro solidphase extraction ( $\mu$-SPE)-HPLC-UV and DLLME-gas chromatography (GC)-flame ionization detector (FID). ${ }^{36-40}$ Furthermore, the extraction time required by the present work was shorter than those of other presented methods except for IL-DLLME/UV-Vis. ${ }^{36-40}$ At last, the hydrophobic DES was used as alternative solvent to IL and organic solvents, which has the disadvantages of high price, toxic and hard preparation. By considering these results, the proposed method is sensitive, rapid, efficient and green that can be used for the determination of the parabens in water samples.

\section{Conclusions}

In the present work, a new method for in situ solvent formation microextraction based on DL-menthol-based DES was developed for the extraction of parabens. A 
Table 4. Comparison of the proposed method with other published microextraction methods

\begin{tabular}{lccccc}
\hline Method & Extraction time / min & Extraction solvent & Linear range / $\left(\mathrm{ng} \mathrm{mL}^{-1}\right)$ & LOD / $\left(\mathrm{ng} \mathrm{mL}^{-1}\right)$ & Reference \\
\hline DLLME-UHPLC-UV & 10 & trichloromethane & $0.63-25$ & $2.5-22$ & 36 \\
DLLME-GC-FID & 10 & 1-octanol & $20-30000$ & $5-15$ & 37 \\
IL-DLLME/UV-Vis & 5 & {$[$ Hmim][PF $]$} & $10-5000$ & $4.2-4.8$ & 38 \\
$\mu$-SPE-HPLC-UV & 40 & - & $1-500$ & $0.08-0.4$ & 39 \\
SDME-GC-MS & 40 & hexyl acetate & $0.05-5$ and 0.1-10 & $1-15$ & 40 \\
In situ DES-LLME & 5 & DES-5 & $3-1000$ & $0.6-0.8$ & this study \\
\hline
\end{tabular}

LOD: limit of detection; DLLME: dispersive liquid-liquid microextraction; UHPLC: ultra high-performance liquid chromatography; GC: gas chromatography; FID: flame ionization detector; IL: ionic liquid; Hmim: 1-hexyl-3-methylimidazolium; $\mu$-SPE: micro solid-phase extraction; HPLC: high-performance liquid chromatography; SDME: single drop microextraction; MS: mass spectrometry; DES: deep eutectic solvent; LLME: liquid-liquid microextraction.

green, environmentally friendly and water-immiscible DES (DES-5) was synthesized in an aqueous solution and it was simultaneously used for parabens extraction. The developed method has certain advantages such as the absence of emulsifier, short extraction time, use of green extraction solvent and high extraction efficiency. The method provided good analytical features, such as low LODs and LOQs, acceptable linear concentration range and good precision. The present work provides an alternative method for determination of parabens in aqueous samples.

\section{Supplementary Information}

Supplementary data are available free of charge at http://jbcs.sbq.org.br as PDF file.

\section{Acknowledgments}

This research was financially supported by the National Natural Science Foundation of China (Grant No. 41463010), the Applied Basic Research Programs of Yunnan Science and Technology Department (Grant No. 2015FD087) and the Projects of Yunnan Education Department (2017ZDX048).

\section{References}

1. Bredin, J.; Davin-Régli, A.; Pagés, J. M.; J. Antimicrob. Chemother. 2005, 55, 1013.

2. Passone, M. A.; Resnik, S. L.; Etcheverry, M. G.; J. Appl. Microbiol. 2005, 99, 682.

3. Ramos-Payan, M.; Maspoch, S.; Llobera, A.; Talanta 2017, $165,496$.

4. Darbre, P. D.; Harvey, P. W.; J. Appl. Toxicol. 2014, 34, 925.

5. Routledge, E. J.; Parker, J.; Odum, J.; Ashby, J.; Sumpter, J. P.; Toxicol. Appl. Pharmacol. 1998, 153, 12.

6. Canosa, P.; Rodriguez, I.; Rubio, E.; Negreira, N.; Cela, R.; Anal. Chim. Acta 2006, 575, 106.
7. Tahan, G. P.; Santos, N. K. S.; Albuquerque, A. C.; Martins, I.; Regul. Toxicol. Pharmacol. 2016, 79, 42.

8. Moreta, C.; Tena, M.-T.; Kannan, K.; Environ. Res. 2015, 142, 452.

9. Saraji, M.; Mirmahdieh, S.; J. Sep. Sci. 2009, 32, 988.

10. Diaz-Alvarez, M.; Turiel, E.; Martin-Esteban, A.; Int. J. Environ. Anal. Chem. 2013, 93, 727.

11. Wei, H.; Yang, J.; Zhang, H.; Shi, Y.; J. Sep. Sci. 2014, 37, 2349.

12. Shen, X.; Liang, J.; Zheng, L.; Lv, Q.; Wang, H.; J. Sep. Sci. 2017, 40, 4385.

13. Farajzadeh, M. A.; Mogaddam, M. R. A.; Ghorbanpour, H.; J. Chromatogr. A 2014, 1347, 8.

14. Wen, X.; Fan, M.; Yang, D.; Li, M.; Li, C.; Zhang, Y.; Anal. Methods 2013, 5, 6030.

15. Abbott, A. P.; Boothby, D.; Capper, G.; Davies, D. L.; Rasheed, R. K.; J. Am. Chem. Soc. 2004, 126, 9142.

16. Abbott, A. P.; Capper, G.; Davies, D. L.; Rasheed, R. K.; Tambyrajah, V.; Chem. Commun. 2003, 7, 70.

17. Zhang, Q.; Vigier, K. D. O.; Royer, S.; Jérôme, F.; Chem. Soc. Rev. 2012, 41, 7108.

18. Leron, R. B.; Li, M.-H.; Thermochim. Acta 2013, 551, 14.

19. Zhao, B.-Y.; Xu, P.; Yang, F.-X.; Wu, H.; Zong M.-H.; Lou, W.-Y.; ACS Sustainable Chem. Eng. 2015, 3, 2746.

20. Habibi, E.; Ghanemi, K.; Fallah-Mehrjardi, M.; DadolahiSohrab, A.; Anal. Chim. Acta 2013, 762, 61.

21. Florindo, C.; Oliveira, M. M.; Branco, L. C.; Marrucho, I. M.; J. Mol. Liq. 2017, 247, 441.

22. Ferrone, V.; Genovese, S.; Carlucci, M.; Tiecco, M.; Germani, R.; Preziuso, F.; Epifano, F.; Carlucci, G.; Taddeo, V. A.; Food Chem. 2018, 245, 578.

23. Moghadam, A. G.; Rajabi, M.; Asghari, A.; J. Chromatogr. B 2018, $1072,50$.

24. Cunha, S. C.; Fernandes, J. O.; TrAC, Trends Anal. Chem. 2018, $105,225$.

25. An, J.; Trujillo-Rodríguez, M. J.; Pino, V.; Anderson, J. L.; J. Chromatogr. A 2017, 1500, 1.

26. Liu, L.; Zhu, T.; Anal. Methods 2017, 9, 4747. 
27. Zeng, H.; Qiao, K.; Li, X.; Yang, M.; Zhang, S.; Lu, R.; Li, J.; Gao, H.; Zhou, W.; J. Sep. Sci. 2017, 40, 4563.

28. Aydin, F.; Yilmaz, E.; Soylak, M.; Food. Chem. 2018, 243, 442.

29. Lamei, N.; Ezoddin, K.; Abdi, M.; Talanta 2017, 165, 176.

30. Panhwar, A. H.; Tuzen, M.; Kazi, T. G.; Talanta 2018, 178, 588.

31. Zhu, S.; Zhou, J.; Jia, H.; Zhang, H.; Food Chem. 2018, 243 , 351.

32. Florindo, C.; Branco, L. C.; Marrucho, I. M.; Fluid Phase Equilib. 2017, 448, 135.

33. Ge, D.; Zhang, Y.; Dai, Y.; Yang, S.; J. Sep. Sci. 2018, 41, 1635.

34. Farajzadeh, M. A.; Mogaddam, M. R. A.; Feriduni, B.; RSC Adv. 2016, 6, 47990.

35. Fume, B. H.; Lanças, F. M.; J. Chromatogr. A 2017, 1487, 64.
36. González-Hernández, P.; Pina, V.; Ayala, J. H.; Afonso, A. M.; Anal. Methods 2015, 7, 1825.

37. Farajzadeh, M. A.; Djozan, D.; Bakhtiyari, R. F.; Talanta 2010 , 81,1360 .

38. Khani, R.; Ghasemi, J. B.; Shemirani, F.; Spectrochim. Acta, Part A 2014, 122, 295.

39. Ara, K. M.; Pandidan, S.; Aliakbari, A.; Raofie, F.; Amini, M. M.; J. Sep. Sci. 2015, 38, 1213.

40. Sarako, M.; Mirmahdieh, S.; J. Sep. Sci. 2009, 32, 988.

Submitted: October 23, 2018

Published online: January 30, 2019 